ePässe übernommen. Der Test fand vom 21. - 23. 05. 2013 im Rahmen der Security Document World (SDW) Konferenz und Ausstellung in London statt.

Der letzte große Test dieser Art wurde 2008 in Prag durchgeführt. In diesem Jahr wird der Fokus des Tests auf die Konformität zum neuen Sicherheitsstandard SAC, Supplemental Access Control, gelegt. Dieser Mechanismus wurde von der ICAO (Internationale zivile Luftfahrt Organisation) zum neuen Standard mit Wirkung ab 2015 definiert und stellt für die nächsten 10 bis 20 Jahre einen noch höheren Schutz bei der Zugriffsberechtigung auf Passdaten sicher. Ab 2015 werden somit ausschließlich elektronische Reisedokumente mit diesem Standard ausgegeben.

Der Interoperabilitätstest wurde von secunet spezifiziert und ausgearbeitet und bietet Herstellern von Identitätsdokumenten sowie Anbietern und Integratoren von Prüfsystemen die Chance, frühzeitig vor Inkrafttreten des neuen Mechanismus ihre Produktions- und Prüfverfahren entsprechend zu testen. secunet wurde von Science Media Partners, dem Veranstalter der SDW, mit der Durchführung und Leitung des dreitägigen Testverfahrens beauftragt, an dem über 30 namhafte Passhersteller und Lieferanten von Passlesegeräten aus aller Welt teilnahmen.

\section{KOBIL: iDtoken für den nPA in allen Postbank Finanzcentern erhältlich}

Der KOBIL iDtoken kann über den Standard USB Port an jeden Computer und jedes Notebook angeschlossen werden. Das neue Produkt von KOBIL ist kompatibel mit dem neuen Personalausweis und durch das Bundesamt für Sicherheit in der Informationstechnik (BSI) zertifiziert. Der Nutzer kann mithilfe des KOBIL iDtoken in Verbindung mit seinem neuen Personalausweis (nPA) bzw. elektronische Aufenthaltstitel (eAT) u. a. Behördengänge und Ausweisnachweise bequem online erledigen, seinen Wohnort bestätigen und mit allen Optionen verwenden. Man hat Zugang zu Diensten mit Altersbeschränkung und kann Formulare automatisch ausfüllen. Die DATEV realisiert über den iDtoken den elektronischen Lohnzettel. Dies sind nur einige von vielen möglichen Anwendungen. Der Kartenleser speichert keinerlei benutzerspezifische Informationen.

Weitere Informationen auf http://www.kobil.com/

\section{IT-Sicherheit als Mittelstandsthema: Fallbeispiel „Hotellerie“}

Der Bundesverband IT-Sicherheit e.V. (TeleTrusT) stellt exemplarisch am Beispiel des laufenden Projektes "IT-Sicherheit in der Hotellerie" heraus: Sensibilisierung für IT-Sicherheit funktioniert branchenspezifisch.

Beherbergungsbetriebe mit überwiegend klein- und mittelständischer Prägung bilden in Deutschland eine bedeutende wirtschaftliche Größe. Durch den geschäftstypischen Umgang mit personenbezogenen Daten und die inzwischen üblichen IT-Anwendungen sind sie in besonderer Weise Thema für IT-Sicherheit bzw. potentielles Angriffsziel. Schadensfälle bedeuten wirtschaftliche Nachteile, haftungsrechtliche Probleme und Rufschaden gleichermaßen. Der IT-Sicherheitsaspekt wird in der Hotelleriebranche nicht selten unterschätzt. Einerseits steigt die Erwartungshaltung der Gäs- te z.B. hinsichtlich Online-Buchungsmöglichkeiten, WLAN-Verfügbarkeit und bargeldlosen Bezahlsystemen, andererseits erhöht sich dadurch das Gefährdungspotential und damit die Haftungsrisiken.

TeleTrusT informiert Betreiber von Beherbergungsstätten auf praxisgerechte Weise und motiviert zu Vorbeugungsmaßnahmen. Das Projekt findet in Kooperation mit dem für die Branche maßgeblichen Hotelverband Deutschland (IHA) und mit Unterstützung der Task Force "IT-Sicherheit in der Wirtschaft" des Bundeswirtschaftsministeriums statt. Diese Dreieckskonstruktion bewährt sich als Kombination aus branchenspezifischem Know-how und politischer Unterstützung und ist für gleichartige Aufklärungskampagnen empfehlenswert.

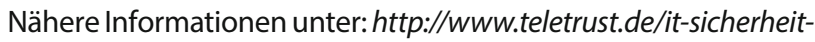
in-der-hotellerie/

\section{LfDI RLP: Datenschutz im Hotelgewerbe}

Datenschutz muss nicht nur im Internet und bei Google, Facebook $\&$ Co. durchgesetzt werden, sondern auch in unserem nicht-digitalen Alltag. Das gilt auch wenn wir dienstlich oder privat den einen oder anderen Tag unterwegs sind und im Hotel übernachten.

Auch dort werden Daten erhoben, gespeichert und genutzt und zwar nicht zu knapp! Es beginnt mit der Erhebung von Meldedaten beim Check-In und endet bei ausgefeilten Kundenprofilen, die gerade große Hotelketten über ihre Gäste anlegen. Manches läuft dabei schief und verletzt die Datenschutzregeln. Nicht ohne Grund ging der Big Brother Award 2007 in der Kategorie Verbraucherschutz an die internationalen Hotelketten in Deutschland.

Auf Grundlage einer mit dem DEHOGA Rheinland-Pfalz durchgeführten Umfrage bei Hotelbetrieben im Land sowie mit den Erkenntnissen aus mehr als 100 Vor-Ort-Kontrollen bei kleinen und mittleren Hotelbetrieben sowie bei allen 19 in Rheinland-Pfalz ansässigen Hotelketten hat sich der rheinland-pfälzische Landesbeauftragte für den Datenschutz und die Informationsfreiheit nun einen Überblick über die Situation des Datenschutzes im Hotelgewerbe verschafft.

Das Resümee von LfDI Edgar Wagner ist zwiespältig: „Viele Hotelbetriebe haben große Anstrengungen unternommen, ihren Kunden auch in Sachen Datenschutz ein guter Gastgeber zu sein. Dennoch sind Probleme bei der weit überschießenden Erfassung von Meldedaten, beim Einsatz von Kreditkarten, bei der Erstellung sensibler Kundenprofile und beim Thema Datensicherheit klar erkennbar", so Wagner. „Hier muss nachgebessert werden!"

Zu diesem Zweck hat der LfDI eine Orientierungshilfe „Datenschutz im Hotelgewerbe" erstellt, die Hoteliers Anleitung zur Bewältigung ihrer "datenschutzrechtlichen Hausaufgaben" und den Hotelgästen praktische Tipps zum Schutz ihrer persönlichen Daten geben soll. Diese Orientierungshilfe wurde am 30.04.2013 - nachdem die Beteiligten angehört worden sind - veröffentlicht und ist im Internetangebot des Landesbeauftragten abrufbar: http://www. datenschutz.rlp.de/downloads/oh/Datenschutz_Hotel.pdf

\section{nPA-BOX: Bayerische Konzeptstudie zum Datensafe im Internet}

Die nPA-Box ist eine sichere Cloud-Lösung zur Speicherung von Daten im Internet. Für die Vertraulichkeit der Daten sorgt die elek- 